\title{
TRADUCCIONES AL CATALÁN Y EXILIO FRANQUISTA ${ }^{1}$
}

\author{
Montserrat Franquesa Gòdia \\ Universidad Autónoma de Barcelona, Facultat de Traducció i \\ Interpretació de la Universidad. Barcelona, España.
}

\begin{abstract}
Resumen: Después de tres años de guerra civil en España, la victoria del dictador Francisco Franco en 1939 supuso el exilio para miles de ciudadanos y muchos intelectuales catalanes tuvieron que abandonar el país. La lengua catalana quedó prohibida en el ámbito público y la producción editorial en catalán también pasó a ser, oficialmente, prohibida. A pesar de las dificultades, algunos traductores prosiguieron su labor en el exilio. Palabras-clave: Traducción catalana. Exilio. Dictadura franquista.
\end{abstract}

\section{CATALAN TRANSLATIONS IN EXILE UNDER FRANCO'S DICTATORSHIP}

Summary: After three years of civil war in Spain, Franco's victory in 1939 meant exile for thousands of citizens, and most Catalan intellectuals had to leave the country. The Catalan language was banned in the public domain and publishing in Catalan became officially forbidden. However, despite the difficulties, some translators continued their work in exile.

Keywords: Catalan translation. Exile. Franco's dictatorship

La victoria en 1939 de los militares sublevados y el establecimiento de la dictadura del general Francisco Franco después de

\footnotetext{
${ }^{1}$ Este artículo se inscribe en el Grup d'Estudi de la Traducció Catalana Contemporània (GETCC) (2014, SGR 285), reconocido por la Agència de Gestió i Ajuts Universitaris de la Generalitat de Catalunya y en el proyecto «La traducción catalana contemporánea: censura y políticas editoriales, género e ideología (1939-2000)», número de referencia FFI201452989-C2-1-P financiado por el Ministerio de Economía y Competitividad.
} 
tres años de guerra civil en España tuvieron como consecuencia inmediata que una parte importante de los intelectuales catalanes emprendieran el camino del exilio. No existe un censo de los expatriados, pero cuando estalló el conflicto, en 1936, Catalunya tenía censados 2.917.851 habitantes y se calcula que el volumen de los que se fueron en 1939 puede oscilar entre 80.000 y 100.000 personas, entre los cuales alrededor de un $20 \%$ correspondería a intelectuales y profesionales liberales (Díaz 1991). La mayoría se dirigió primero hacia Francia, para luego continuar hacia otros destinos. En muchos casos la huida les llevó hasta el otro lado del Atlántico, principalmente a México, pues el presidente Lázaro Cárdenas había ofrecido asilo político a los republicanos españoles desde 1936. Españoles y catalanes también llegaron a Argentina, Chile, República Dominicana, Estados Unidos o Canadá tras múltiples peripecias y vicisitudes, testimoniadas para la posteridad en los relatos de viajes, cartas, memorias o dietarios (Miravitlles 2009). Se trataba de una diáspora que huía por razones ideológicas y en el caso de Catalunya del genocidio cultural y lingüístico (Benet 1995) que perpetró el régimen dictatorial de Franco hasta 1975 y que sigue siendo, aún hoy en día, poco revisado y estudiado.

Las investigaciones de Teresa Férriz permitieron la publicación del estudio La edición catalana en México, donde se aportan datos concretos sobre el crecimiento de las publicaciones en catalán de la comunidad de exiliados dentro de la cual se encontraban buena parte de los dirigentes políticos y de los intelectuales catalanes. Entre 1939 y 1947 vieron la luz en México más de doscientos títulos (Férriz 1998, 15). Durante esos años, México se convirtió en el centro de la cultura catalana y aún en 1947 se consideraba que la Catalunya real era la de los exiliados, pues no fue un sector social o una clase determinada la que tuvo que cruzar la frontera en 1939, sino que, parafraseando a Josep Navarro Costabella, fue Catalunya entera la que fue al exilio. La mayoría de escritores, profesores y traductores de la época se vieron obligados a abandonar el país: Josep Carner, Pere Calders, Ferran Canyameres, Lluís Ferran de Pol, Ventura Gassol, Cèsar August Jordana, Joan Oliver, Josep 
Pous i Pagès, Joan Puig i Ferreter, Carles Riba, Mercè Rodoreda, Pau Romeva, Antoni Rovira i Virgili, Josep M. de Sagarra, Ferran Soldevila, Rafael Tasis, por citar algunos. Otros murieron durante los tres años de guerra: Joaquim Balcells, Carles Capdevila, Pere Coromines, Alfons Maseras, Lluís Segalà o Andreu Nin. Para muchos, la solución pasó por dejar de escribir o de traducir para siempre. Así pues, la vida cultural catalana quedó silenciada a la vez que la dictadura de Franco intentaba relegar las lenguas diferentes al castellano a una anécdota folklórica, buscando la destrucción del público lector en gallego, vasco y catalán. La supremacía de una sola lengua y cultura, la castellana, iba en consonancia con la ideología fascista e intolerante con las minorías lingüísticas.

Ante este panorama, no resulta difícil comprender que las penurias de los exiliados catalanes, escritores o traductores, para continuar ejerciendo su oficio, resultaran enormes y que las traducciones al catalán que produjo la diáspora fueran escasas.

\section{¿Qué se tradujo en el exilio franquista?}

Albert Manent, en el libro de referencia sobre la situación de las letras catalanas en el exilio franquista, se refería a la insignificante cantidad de traducciones, pues los esfuerzos para la supervivencia de la literatura catalana debían dar prioridad a las obras originales y dejar las versiones de autores extranjeros para tiempos mejores (Manent 1976, 220). Manent enumera siete traducciones al catalán, tres de las cuales se editaron en Perpiñán por parte de la editorial Proa en los años cincuenta y sesenta: L'hereu de Ballantrae (1953) de R. L. Stevenson traducido por Cèsar-August Jordana; L'estany del diable (1955) de George Sand, traducción de Just Cabot y Homes i ratolins de John Steinbeck (1964), por Manuel de Pedrolo. En cuanto a poesía, Manent destaca la Antologia de la lírica nord-americana que vio la luz en México en 1951, elaborada por Agustí Bartra. Resulta evidente que el balance total de las traducciones al catalán a lo largo de treinta y cinco años sea mínimo, como expone Montserrat 
Bacardí en un primer estudio panorámico, resultando un promedio de una versión cada cinco años (Bacardí 2009, 10). Cabe recordar que el franquismo estableció unas normas muy restrictivas por lo que respecta a las traducciones, en la medida que constituyen la base para la transmisión de ideologías (Gallofré 1991, 380), de manera que la única posibilidad de publicar versiones de obras extranjeras se encontraba más allá de las fronteras. En este sentido, la constatación de la existencia de traducciones publicadas, de manera completa o fragmentaria, en las revistas de los círculos intelectuales catalanes de América, ha abierto un campo de investigación, sobre todo a partir de los índices completos de las revistas culturales más importantes del exilio en México (Férriz 2009): La Nostra Revista (1946-1954), La Nova Revista (1955-1958), Pont Blau (1952-1963), Quaderns de l'Exili (1943-1947) i Xaloc (1964-1981). ${ }^{2}$ En cualquier caso, resulta verdaderamente sorprendente saber que sin un público lector y fuera del ámbito lingüístico natural, traductores como Agustí Bartra, Josep Carner, Joan Oliver, Francesc Vidal Jové o Ramon Xuriguera hicieran versiones al catalán de Whitman, Eliot, Joyce, Dickens, Valéry, Molière o Flaubert. Sirva como ejemplo la traducción por parte de Carles Riba de un cuento de los hermanos Grimm en la revista Full Català de México, especificando «Perquè el vostre infant llegeixi en català», ${ }^{3}$ prueba de la necesidad de las comunidades catalanas de América de tener lecturas infantiles que permitieran, de alguna manera, la transmisión generacional de la lengua materna también en letra impresa (Manent 1985).

\section{¿Cómo y por qué traducir al catalán en el exilio?}

En el apartado dedicado a los traductores, Teresa Férriz afirmaba que en un primer momento y también después, los desterrados

\footnotetext{
${ }^{2}$ Véase http://lletra.uoc.edu/exili/cat/revistes/lanostrarevista/index.html por lo que respecta a las revistas más significativas del exilio catalán y que aparecieron con regularidad.

${ }^{3}$ «Para que vuestro hijo lea en catalán». Full Català, vol. II, n ${ }^{\circ} 6$, marzo 1942. México.
} 
encontraron en las traducciones un modus vivendi nada despreciable (Férriz 1998, 114). Un ejemplo de ello fue el mercado mexicano, en auge en los años cuarenta, donde el aumento del público lector consolidó las ediciones del Fondo de Cultura Económica, con un amplio catálogo y con proyección por toda América Latina. Entre los traductores catalanes del Fondo se encuentran intelectuales de la talla de Joan Roura, Eduard Nicol, Alfred Pereña, Joaquim Xirau y un largo etcétera. Los encontramos traduciendo al español, pero no al catalán. Fue el caso de no pocos intelectuales catalanes que, adaptados a una nueva realidad, se dedicaron, por necesidades económicas o porque ése era realmente su oficio, a traducir al castellano. El dominio de idiomas les ayudó a sobrevivir ante una realidad completamente nueva pero tuvieron que pasar, de alguna manera, a tener una doble vida lingüística. Traducir al catalán y a miles de quilómetros de Cataluña resultaba, a priori, imposible. Sin embargo, y como citábamos anteriormente, la diáspora catalana fue capaz de publicar versiones de autores extranjeros en su propia lengua dirigidas al lector que mantenía el contacto con su cultura materna gracias a los centros culturales, como el Orfeó Català de México o el Casal Català de Buenos Aires.

\section{Los artífices de las versiones al catalán: cuatro nombres propios}

Tres fueron los traductores más significativos obligados al exilio franquista, de generaciones diferentes y que habían sido prolíficos antes de la guerra: Josep Carner i Puig-Oriol (1884-1970), Cèsar August Jordana (1893-1958) y Agustí Bartra (1908-1982).

Josep Carner, poeta y traductor, el que fuera secretario de la Sección Filológica de l'Institut d'Estudis Catalans y director de la ambiciosa Editorial Catalana entre 1918 y 1921, se exilió en México entre 1939 y hasta 1945, donde tradujo abundantemente al español para el Fondo de Cultura Económica y otras editoriales, después se instaló en Bruselas (hasta su muerte, el 4 de junio 
de 1970), sin dejar nunca de colaborar con los proyectos de los exiliados. De sus primeras traducciones destacan El somni d'una nit d'estiu y Les alegres comares de Windsor de Shakespeare (de 1908 y 1909, respectivamente), El malalt imaginari de Molière (1909), Les aventures de Tom Sawyer de Mark Twain (1918) y las Faules de La Fontaine (1921), por citar algunos de los treinta y dos títulos de versiones que vieron la luz antes del conflicto civil. Después de la guerra, tan sólo publicó tres traducciones al catalán: David Copperfield de Charles Dickens (1964), Càntic del sol de Francesc d'Assís (1976) y Assaigs de Francis Bacon (1976). Es evidente que la producción de versiones al catalán de Carner sufrió las consecuencias del destierro. Sin embargo, en 1944, en la revista Lletres de México, publicación periódica que había fundado con Agustí Bartra, aparecieron una serie de aforismos bajo el título « De l'art de traduir », donde resume su ideario sobre el arte de traducir. Se trata de una serie de aforismos o máximas que se añaden a la reflexión teórica sobre la traducción en el mundo occidental desde que lo hiciera Cicerón. Carner consideraba que el buen traductor debe evitar la desnaturalización resultante de un literalismo excesivo y traducir libremente e impregnar con la propia personalidad la obra traducida. Asimismo, Carner expresaba los beneficios que los escritores y la cultura nacional obtienen gracias a las traducciones. También había reflexionado sobre la traducción Cèsar August Jordana, pues en 1938 había publicado «L'art de traduir » en la Revista de Catalunya (Jordana 1938), un ensayo fundamental para la historia y teoría de la traducción que incluye ejemplos fruto de su experiencia como traductor de la lengua inglesa.

Cèsar August Jordana, conocido por ser el traductor más prolífico de Shakespeare al catalán antes de la guerra, llegó en enero de 1940 a Chile con su familia y las de otros catalanes que viajaban en el mismo barco, que desde Marsella hizo escala en Casablanca y Montevideo hasta atracar en Buenos Aires: Francesc Trabal, Joan Oliver y Xavier Benguerel, parte del llamado «núcleo de la Institució de les Lletres Catalanes» por su prestigio intelectual. 
En Santiago de Chile Jordana empezó a ganarse la vida haciendo traducciones y en 1945 se trasladó a Buenos Aires. Los primeros años no fueron nada fáciles. Según leemos en su correspondencia con los amigos más íntimos, tuvo que trabajar muy duro para salir adelante, traduciendo a una lengua que no era la propia, con la sensación de que se estaba olvidando de la lengua materna. ${ }^{4} \mathrm{Al}$ igual que Josep Carner, el número de traducciones al catalán, más de veinticinco antes del conflicto, se reducen a tan sólo dos en los años del exilio: L'hereu de Ballantrae, citado anteriormente, y un Ivanhoe de Walter Scott que apareció en Barcelona sin fecha en el pie de imprenta. Otras dos traducciones al catalán del alemán permanecen inéditas y suponemos que fueron las últimas que realizó, con el objetivo de poner a prueba el dominio del idioma materno. Los manuscritos de Die Räuber de Schiller y un inacabado Egmont de Goethe se conservan inéditos en el Archivo Nacional de Catalunya (Franquesa 2015).

Agustí Bartra también vio truncada su fecunda carrera literaria por la guerra civil y el exilio. Después del paso por tres campos de concentración en Francia, por la República Dominicana y Cuba, se instaló en México hasta 1970, junto a su esposa, como ya hemos mencionado, la también escritora y traductora Anna Murià. A lo largo de treinta años se dedicaron a la traducción de todo tipo de obras del inglés y francés al español. Bartra recibió en 1949 una beca de la Fundación Guggenheim para traducir una muestra de poesía norteamericana, que apareció en México en 1952 con el título Antología de la lírica norteamericana y que un año antes había conseguido publicar en catalán. De esta manera, vieron la luz en catalán obras de autores completamente inéditos, desde Walt Whitman hasta los poetas más jóvenes del momento, razón por la cual se le considera el introductor de la modernidad internacional en las letras catalanas, realizando una doble tarea de antólogo y de traductor (Abrams 2009, 69). Bartra se incluye también como

${ }^{4}$ En una carta a Agustí Bartra desde Santiago de Chile el 8 de abril de 1941 (conservada en el Fons C. A. Jordana, número 680, Arxiu Nacional de Catalunya) confesaba tener la sensación de estar perdiendo la lengua materna, de tanto leer y escribir solo en castellano.

Cad. Trad., Florianópolis, v. 38, $\mathbf{n}^{0}$ 1, p. 36-46, jan-abr, 2018 
un caso de auto-traducción, pues tradujo parte de su propia obra al español después de haberla producido en catalán. Sin embargo, el gran mérito de Bartra es haber sido el primer traductor de T.S. Elliot al catalán: The waste land vio la luz en 1977 en Barcelona como La terra eixorca (Ruiz 2003, 57).

En los años del tardofranquismo, el traductor al catalán más prolífico en el exilio fue Jordi Arbonès, que se trasladó a Argentina en 1956, donde permaneció hasta su muerte en 2001. Se propuso vivir de la traducción, de manera que ejerció su oficio de manera incansable, primero al castellano y después, a partir de 1966 y sobre todo a raíz de sus colaboraciones con el Casal Català, al catalán. La lista de autores ingleses y norteamericanos es larguísima: Jane Austen, Saul Bellow, Karen Blixen, Paul Bowles, Agatha Christie, Charles Dickens, Vladimir Nabokov, Tolkien, Gore Vidal, etc. Obtuvo diversos premios y reconocimientos, como el Premi Nacional de Traducció en 1991, que otorga la Institució de les Lletres Catalanes, por la traducción d'Una història de dues ciutats de Charles Dickens. La Universitat Autònoma de Barcelona es la depositaria de su legado desde la creación en 2003 de la Càtedra de Traducció Jordi Arbonès, que pone a disposición de los investigadores un sinfín de documentación aún por explorar.

\section{A modo de conclusión}

En la última página de su memoria personal del éxodo, fechada el 5 de febrero de 1939, el político y escritor Antoni Rovira i Virgili escribió: «Enmig del present desolat i tràgic, poso la meva esperança en els dies que vindran, en el dret que triomfarà, en les llibertats que es restabliran, en la llengua que persistirà» ${ }^{5}$ y depositaba su confianza en los exiliados para que algún día la patria derrotada pudiera resurgir (Rovira i Virgili 2004, 204). Ante la

${ }_{5}$ «Ante el presente desolador y trágico, deposito mi esperanza en los días venideros, en el derecho que triunfará, en las libertades que se restablecerán, en la lengua que persistirá» (Traducción de la autora). 
magnitud del desastre cultural que dejó el franquismo, la intelectualidad de la diáspora consiguió mantener vivas la lengua y la cultura catalanas fuera de sus fronteras naturales. La mayoría tuvo que dedicarse a traducir al español, que aunque dominaran, no era su lengua materna. Traductores como Josep Carner o Cèsar-August Jordana pasaron a no publicar en catalán durante los primeros años de exilio. A pesar de todo, a lo largo de los años cuarenta, en México y sobre todo gracias a las publicaciones periódicas de las comunidades catalanas de América Latina, vieron la luz algunas traducciones esporádicas que ayudaron a mantener viva la lengua.

España vivió en un completo vacío por lo que respecta a la llegada de obras extranjeras prácticamente hasta 1966, cuando se promulgó la Ley de Prensa e Imprenta bajo el mandato del ministro Manuel Fraga Iribarne. En la inmediata postguerra, en 1942, la censura sólo concedió permiso de traducción a las publicaciones de los clásicos grecolatinos de la colección Fundació Bernat Metge, cuyo mecenas, Francesc Cambó, se había posicionado a favor del nuevo régimen (Franquesa 2016). A partir de 1947, cuando el final de la Segunda Guerra Mundial provocó una postura más abierta del gobierno dictatorial, se concedió la publicación de algunas obras con el nihil obstat de los censores. Por esas fechas, traductores catalanes ya habían publicado interesantes reflexiones teóricas sobre el oficio del traductor (Jordana en 1938 y Carner en 1944). Por otro lado, la Antologia de lírica nord-americana de Agustí Bartra vivificaba la lengua catalana aportando la modernidad a la cultura catalana fuera de sus fronteras. No nos parece exagerado afirmar que la traducción, gracias al esfuerzo de sus artífices, también contribuyó a la evolución de una cultura y a mantener vivo el espíritu de una lengua, la catalana, que una dictadura fascista pretendió condenar a la extinción. 


\section{Referencias}

Abrams, Sam. «Quelcom neix en tota mort... ». Quaderns, Revista de Traducció 16 (2009): 67-73.

Bacardí, Montserrat. «La traducció catalana a l'exili. Una aproximació», Quaderns, Revista de Traducció 16 (2009): 9-21.

Benet, Josep. L'intent franquista de genocidi cultural contra Catalunya. Barcelona: Publicacions de l'Abadia de Montserrat, 1995.

Díaz Esculies, Daniel. El catalanisme polític a l'exili (1939-1959). Barcelona: Editorial La Magrana, 1991.

Férriz Roure, Teresa. La edición catalana en México. Jalisco: El Colegio de Jalisco, 1998.

. Escriptors i revistes catalanes de l'exili. Barcelona: UOC, 2009.

Franquesa, Montserrat. «Dos traducciones inéditas de C.A. Jordana en el exilio: Die Räuber de Schiller y Egmont de Goethe». Interacciones. Wechselwirkungen. Studien zur romanischen Sprachwissenschaft und interkultureller Kommunikation, 103. Frankfurt am Main: Peter Lang Edition (2015): 369-387.

Franquesa, Montserrat. «La Fundació Bernat Metge sota el franquisme. L'escenari de la represa (1939-1942)». Literatura catalana contemporània: crítica, transmissió textual $i$ didàctica. Barcelona: Societat catalana de llengua $\mathrm{i}$ literatura-UVic-UCde Catalunya (2016): 49-70.

Gallofré, Maria Josep. L'edició catalana i la censura franquista (1939-1951). Barcelona: Publicacions de l'Abadia de Montserrat, 1991.

Jordana, Cèsar August. "L'art de traduir ». Revista de Catalunya, 88 (julio de 1938): 357-370. 
Manent, Albert. La literatura catalana a l'exili. Barcelona: Curial, 1976. . «Llibre en català, per a infants, a Mèxic». Serra d'Or 1 (1985): 69.

Miravitlles, Jaume. D'Europa a Amèrica: dietari d'exili (1941-1945). Barcelona: Proa, 2009.

Rovira i Virgili, Antoni. Els darrers dies de la Catalunya republicana. Memòries sobre l'èxode català. Barcelona: A Contra Vent Editors, 2014.

Ruiz Casanova, José Francisco. «Voces de la razón muda. Dos traductores del exilio: Agustí Bartra y Juan Ortega Costa». Vasos Comunicantes 27 (2003): 51-59.

Recebido em: 26/08/2017

Aceito em: 15/10/2017

Publicado em janeiro de 2018

Montserrat Franquesa Gòdia é licenciada en Filología Clásica y en Filología Germánica por la Universidad de Barcelona y Doctora en Teoría de la Traducción por la Universidad Autónoma de Barcelona. Profesora de Historia de la Traducción en la Facultat de Traducció i Interpretació de la Universidad Autónoma de Barcelona e investigadora del GETCC, Grup d'Estudi de la Traducció Catalana Contemporània (http://grupsderecerca.uab.cat/getcc/). Barcelona, España. E-mail: montserrat.franquesa@uab.cat 